\title{
ANÁLISE DE FATORES DE RISCO PARA BAIXO PESO E PREMATURIDADE EM RECÉM-NASCIDOS DE GESTANTES ADOLESCENTES EM FEIRA DE SANTANA - BA NO PERÍODO 2006 A 2012.
}

\author{
$\underline{\text { Rebeca Ribeiro Ferreira }}{ }^{1}$; Maria Conceição Oliveira Costa $^{2}$; Naysa Farias Barros ${ }^{3}$ e \\ André Henrique do Vale de Almeira ${ }^{4}$ \\ 1. Bolsista PROBIC/UEFS, Graduando em Psicologia, Universidade Estadual de Feira de Santana, e-mail: \\ rebeca.ribeiro20.rr@gmail.com \\ 2. Orientador, Departamento Saúde, Universidade Estadual de Feira de Santana, e-mail: oliveiramco69@ gmail.com 3. \\ Participante do núcleo de Estudos e Pesquisas na Infância e Adolescência, graduanda de Enfermagem, Departamento de \\ Saúde, Universidade Estadual de Feira de Santana, e-mail: naysafarias@ gmail.com \\ 4. Participante do Núcleo de Estudos e Pesquisas na Infância e Adolescência, Professor substituto do Departamento de Saúde, \\ Universidade Estadual de Feira de Santana, e-mail: almeida_ahv@ hotmail.com
}

Palavras-chave: Gravidez na Adolescência; Recém-Nascidos; Prematuridade.

\section{INTRODUÇÃO}

A maternidade na adolescência consiste um fenômeno de repercussão mundial e possui inúmeros significados nas diversas culturas e contextos, representando um desafio de saúde pública, visto que pode ocasionar problemas econômicos, familiares, psicossociais e complicações obstétricas que comprometem a saúde materna e do recém-nascido (RN) (CHALEN et al., 2007; SANTOS et al., 2014).

Nessa perspectiva, a gravidez precoce deve ser considerada de risco. Inúmeros aspectos relevantes, tais como o difícil acesso a serviços de saúde, o baixo nível socioeconômico, comportamentos de risco, hábitos e nutrição inadequada, demonstram a necessidade de controle dos diversos fatores associados à evolução e ao desfecho da gestação e condições de saúde do recém-nascido e, consequentemente, uma particular atenção à saúde materna e fetal (MARTINEZ et al., 2011; SANTOS et al., 2014).

Este estudo teve como objetivo avaliar a associação entre a gravidez de adolescentes $\leq 16$ anos e a ocorrência de nascidos vivos de baixo peso e prematuros, com objetivos específicos de analisar as características sociodemográficas de pré-natal e de nascidos vivos de mães adolescentes; avaliar os fatores de risco associados ao baixo peso e a prematuridade em recém-nascidos de mães adolescentes.

Ao considerar que a maternidade nessa fase é um tema de relevância mundial, se justifica a importância de desenvolver pesquisas nessa temática com o intuito de sensibilizar às autoridades para a elaboração de atitudes e programas de prevenção da gravidez precoce e de métodos contraceptivos destinados aos adolescentes. Assim, o interesse na realização desse estudo surgiu devido à experiência como voluntária do Núcleo de Estudos e Pesquisas na Infância e Adolescência, em que houve a oportunidade de conhecer e explorar essa temática. MATERIAL E MÉTODOS OU METODOLOGIA (ou equivalente)

Estudo transversal, foram utilizadas informações das Declarações de Nascidos Vivos dos RN de mães adolescentes ( $\leq 16$ anos) cujos partos ocorreram em Feira de Santana, no período 2006 a 2012. Foram utilizados dados do Sistema de Informação sobre Nascidos Vivos (SINASC), obtidos através do DATASUS, disponibilizados pela $2^{a}$ Diretoria Regional do Estado da Bahia (DIRES) do município. As mães foram divididas por faixa etária < 16 anos, sendo as variáveis classificadas tipo de parto (normal ou cesariano), relacionadas ao RN (peso de nascimento e idade gestacional). Os RN foram classificados segundo a Organização Mundial da Saúde (1995) em de baixo peso (<2500g); de peso insuficiente (2500-2999g) e 
peso adequado ( $>3000 \mathrm{~g}$ ); prematuros ( $<37$ semanas). Os dados foram analisados através do software estatístico stata 10.0, sendo empregada a regressão logística, expressando- se os resultados em razão de odds (OR) com intervalo de confiança (95\%), para a análise multivariada, obtendo-se a força de associação entre as variáveis, ajustadas para os fatores de confundimento, segundo os modelos de análise.

\section{RESULTADOS E/OU DISCUSSÃO (ou Análise e discussão dos resultados)}

No período entre 2006 a 2012, o SINASC de Feira de Santana registrou 19.869 nascidos vivos de mães adolescentes, sendo que as mães $\leq 16$ anos representou 30,2\%. A análise de regressão logística, com controle dos fatores de confundimento, foi sistematizada em três modelos para a faixa etária materna $\leq 16$ anos (Tabelas 1,2 e 3 ): o baixo peso do RN mostrou associação significante com a faixa etária materna $\leq 16$ anos, (OR 1,21); com parto cesariana (OR 1,34) e na interação estatística do pré-natal inadequado e parto cesariana (OR 1,65$)$ (Modelo I); o peso insuficiente do RN também apresentou associação estatística com a faixa etária materna ( $\leq 16$ anos) (OR 1,20); nesse grupo de RN, o parto cesariana e o estado civil solteira mostraram-se como fatores de proteção (OR 0,74 e 0,91, respectivamente) (Modelo II); a prematuridade apresentou associação significante apenas nos casos dos RN de baixo peso (OR 10,77) (Modelo III):o parto cesariana mostrou associação significante no grupo de mães solteiras (OR 1,24), com pré-natal inadequado (OR 1,58) e RN com baixo peso (OR 1,34) (Modelo IV).

Tabela 1. Análise de regressão logística dos fatores associados à ocorrência de RN com baixo peso (Modelo I) e peso insuficiente (Modelo II) ao nascer, entre mães adolescentes na faixa < 16 anos. Registros do SINASC, período 2006-2012, Feira de Santana (BA).

\begin{tabular}{|c|c|c|c|c|c|c|}
\hline \multirow{2}{*}{ Covariável } & \multicolumn{3}{|c|}{ Modelo I (baixo peso) } & \multicolumn{3}{|c|}{ Modelo II (peso insuficiente) } \\
\hline & OR & P-Valor & IC(95\%) & OR & P-Valor & IC (95\%) \\
\hline Idade Materna & 1,214 & 0,000 & $1,129-1,304^{*}$ & 1,201 & 0,000 & $1,117-1,291^{*}$ \\
\hline $\begin{array}{l}\text { Pré-Natal/ } \\
\text { Inadequado }\end{array}$ & 0,778 & 0,114 & $0,570-1,061$ & 0,789 & 0,139 & 0,577-1,079 \\
\hline Cesariana & 1,341 & 0,000 & $1,251-1,436^{*}$ & 0,744 & 0,000 & $0,693-0,797^{*}$ \\
\hline $\begin{array}{c}\text { Estado Civil/ } \\
\text { Solteira }\end{array}$ & 0,924 & 0,057 & $0,852-1,002$ & 0,918 & 0,042 & $0,845-0,996^{*}$ \\
\hline $\begin{array}{l}\text { Pré-Natal } \\
\text { Cesariana }\end{array}$ & 1,654 & 0,039 & $1,026-2,665^{*}$ & 1,582 & 0,061 & $0,978-2,559$ \\
\hline
\end{tabular}

* Estatisticamente significante $(p<0,05)$; os RN foram comparados em grupos, segundo peso de nascimento (peso adequado $x$ baixo peso) e (peso adequado $x$ peso insuficiente).

Tabela 2. Análise de regressão logística dos fatores associados à ocorrência de prematuros (<37 semanas), entre mães na faixa $<16$ anos. Registros do SINASC, período 2006-2012, Feira de Santana (BA).

\begin{tabular}{cccc}
\hline & \multicolumn{3}{c}{ Modelo III } \\
\cline { 2 - 4 } Covariável & OR & P-Valor & IC (95\%) \\
\hline $\begin{array}{c}\text { Peso ao nascer } \backslash\left({ }^{* *} \text { peso }\right. \\
<3000 \mathrm{~g})\end{array}$ & 10,77 & 0,000 & $7,86-14,75^{*}$
\end{tabular}




\begin{tabular}{cccc} 
Idade Materna & 1,17 & 0,274 & $0,87-1,57$ \\
Estado Civil/ Solteira & 0,86 & 0,412 & $0,62-1,21$ \\
Tipo de Parto/ Cesariana & 1,11 & 0,420 & $0,85-1,47$ \\
Escolaridade Materna & 1,48 & 0,103 & $0,92-2,38$ \\
\hline Estatisticamente significante $(p<0,05) ; * *$ & Peso insuficiente + baixo peso.
\end{tabular}

Tabela 3. Análise de regressão logística dos fatores associados à cesariana, entre mães na faixa $<16$ anos. Registros do SINASC, período 2006-2012, Feira de Santana (BA).

\begin{tabular}{cccc}
\hline & \multicolumn{3}{c}{ Modelo IV } \\
\cline { 2 - 4 } Covariável & OR & P-Valor & IC (95\%) \\
\hline Idade Materna & 0,96 & 0,340 & $0,90-1,03$ \\
Estado Civil/ Solteira & 1,24 & 0,000 & $1,16-1,34^{*}$ \\
Pré-Natal/Inadequado & 1,58 & 0,009 & $1,12-2,23$ \\
Peso ao nascer/baixo peso & 1,11 & 0,420 & $0,85-1,47$ \\
Pré-Natal/Insuficiente Idade & 0,77 & 0,242 & $0,50-1,18$ \\
Materna & & & \\
\hline
\end{tabular}

* Estatisticamente significante $(p<0,05)$.

Os achados que mostraram associação estatisticamente significante entre faixa etária materna < 16 anos e RN com baixo peso e peso insuficiente, em relação aos de peso adequado estão de acordo com dados de estudos de distintos contextos e regiões do Brasil (nordeste e sudeste), com registros do SINASC, que evidenciaram maiores prevalências de $\mathrm{RN}$ de baixo peso entre mães das faixas muito jovens (< 16 anos), comparadas às adultas jovens. Os recém-nascidos este grupo de mães apresenta maior tendência para peso insuficiente e baixo peso. Diante destes dados nota-se a influência de fatores como a assistência pré-natal inadequada, ausência do parceiro e a não aceitação da gestação pela família ou companheiro, entre outros, no estado de saúde e bem-estar da gestante adolescente, favorecendo condições adversas ao crescimento e desenvolvimento fetal.

Foram 30,2\% de nascidos vivos de gestantes adolescentes no período e local já citados, dentro do total de 19.869 nascidos vivos de adolescentes de 10 a 19 anos.

$\mathrm{Na}$ análise da regressão logística, constata-se interação estatística do pré-natal inadequado e parto cesariana (OR 1,65). Sabe-se que os serviços de pré-natal têm sido executados de maneira tecnicista e centrado no biologismo, o direcionamento ao qual é feito o pré-natal incide diretamente no processo gestacional e consequentemente na saúde da mãe e do bebê. A importância pré-natal consiste no fator determinante para a evolução normal da gravidez, a qual possui o objetivo de minimizar a morbimortalidade materno-fetal. Quanto melhor sua qualidade, mais favorável será o resultado (MIRANDA et al., 2013). Segundo a Organização Mundial de Saúde (OMS) e o Programa de Humanização no Pré-natal e Nascimento (PHPN), para que o acompanhamento seja considerado adequado deverá ser iniciado no primeiro trimestre e registrar um mínimo de seis consultas (BRASIL, 2005).

Em relação ao baixo peso os achados mostram que a prematuridade é a maior causa da ocorrência de baixo peso ao nascer, apresenta-se como principal fator de mortalidade infantil, pelo fato do recém-nascido imaturo não possuir desenvolvimento completo dos órgãos, como cérebro e pulmões, além de limitação da função renal e imaturidade da função hepática, podendo sofrer sérios comprometimentos. Muitas vezes o risco de partos pré-termos na adolescência está associado ao aumento de infecção subclínica e produção de prostaglandinas devido à imaturidade do útero ou o suprimento de sangue do colo do útero (GRAVENA et al., 2013). 


\section{CONSIDERAÇÕES FINAIS (ou Conclusão)}

Conclui-se que a gestação precoce está associada tanto a fatores biológicos, quanto a outros múltiplos aspectos de ordem psíquica e social. Acerca da condição do parto e dos recém-nascidos dessas mães adolescentes, há maior incidência de prematuridade. Os dados mostram que mães muitos jovens (menores de 16 anos), em comparação com adultas gestantes, são mais susceptíveis e vulneráveis. Essa vulnerabilidade decorre de fatores ligados ao estilo de vida, à condição social e econômica, além dos possíveis fatores biológicos.

O nível socioeconômico implica diretamente no acesso aos serviços de saúde e acompanhamento gestacional e o nível educacional impacta nos comportamentos de risco, nutrição adequada, cuidados e hábitos saudáveis para o desenvolvimento da gestação. A estabilidade destes níveis é fundamental para as jovens gestantes formular habilidades e estratégias para promover o próprio cuidado e do bebê. Além de problemas psicossociais, a gestação causa transformações físicas relacionadas ao ritmo metabólico e hormonal.

As políticas públicas de saúde precisam proporcionar atenção à crianças e adolescentes por se caracterizarem como grupo populacional vulnerável compreendidos em um conjunto de fatores de natureza biológica, epidemiológica, social de risco (GURGEL et al., 2010). Logo, este grupo precisa de atenção para possibilitar o potencial familiar, comunitário e pessoal de cada indivíduo. A educação sexual promove o fornecimento de informações que motiva atitudes responsáveis na vida sexual. Ainda que profissionais da rede saúde tenha o dever de desenvolver estratégias de prevenção e/ou cuidados da gravidez, é o público alvo que aplica estes métodos, por isso deve ser orientado para que tenha capacidade de auto-cuidado físico e psíquico.

\section{REFERÊNCIAS}

BRASIL, Ministério da Saúde. Manual Técnico Pré-Natal e Puerpério Atenção Qualificada e Humanizada. Brasília, 2005

CHALEM, E. et al. Gravidez na adolescência: perfil sóciodemográfico e comportamental de uma população da periferia de São Paulo, Brasil. Cad. Saúde Pública, Rio de Janeiro, v. 23, n. 1, p. 177-186 jan, 2007.

DADOORIAN, Diana. Gravidez na adolescência: um novo olhar. Psicol. cienc. prof. [online]. v. 23, n.1, p.84-91, 2003.

GRAVENA, Angela Andréia França, et al. Idade materna e fatores associados a resultados perinatais. Acta Paulista de Enfermagem.v 26, n. 2, p. 130-5, 2013.

GURGEL, Maria Glêdes Ibiapina et al. Desenvolvimento de habilidades: estratégia de promoção da saúde e prevenção da gravidez na adolescência. Rev. Gaúcha Enferm.

(Online). v. 31, n.4, p.640-646, 2010.

MARTINEZ, E. Z. et al. Gravidez na adolescência e características socioeconômicas dos municípios do Estado de São Paulo, Brasil: análise espacial. Cad. Saúde Pública, Rio de Janeiro, v. 27, n. 5, p. 855-867, mai, 2011.

MIRANDA, Fátima Regina Dias de; TAQUETTE, Stella R.; MONTEIRO, Denise LeiteMaia; BLANCO,Mariangela Nogueira; RODRIGUES, Adriana de Oliveira. Pré-natal na adolescência: uma revisão crítica. Adolescência e saúde, v. 10, n. 1, p. 43-50. 2013. MONTEIRO, Nancy R. de Oliveira. Percursos da Gravidez na Adolescência: Estudo Longitudinal Após uma Década da Gestação. Psicologia: Reflexão e Crítica, v. 23, n. 2, p. 278-288, 2010.

SANTOS, N. L. A. C. et al. Gravidez na adolescência: análise de fatores de risco para baixo peso, prematuridade e cesariana. Ciênc. saúde coletiva [online], v.19, n.3, p.719-726, 2014. 